\title{
EQUILIBRIUM CLUSTER DISTRIBUTIONS OF THE THREE-DIMENSIONAL ISING MODEL IN THE ONE PHASE REGION*
}

\author{
J. MARRO and R. TORAL \\ Departamento de Fisica Teórica, Universidad de Barcelona, Diagonal-647, Barcelona-28, Spain
}

Received 24 January 1983

In final form 28 June 1983

\begin{abstract}
We analyse equilibrium cluster distributions obtained numerically from a ferromagnetic Ising model (simple cubic lattice, 125000 sites and periodic boundary conditions) along the coexistence line and in the one-phase region below $T_{\mathrm{c}}$. We find evidences that the distribution of sizes and energies scales with temperature and external magnetic field giving Binder's droplet exponent $y \approx 4 / 9$. The mean number of incident (interior and exterior) bonds on a cluster of size $l, s_{l}$, seems to behave as $l^{x}$ with $x \approx 9 / 10$ when not far away from $T_{\mathrm{c}}$. We conclude that while the classical nucleation theory may provide an approximate description around $0.59 T_{\mathrm{c}}$, it has to be modified at higher (and lower) temperatures. The Fisher droplet model and the approach by Penrose et al. based on a renormalized fugacity are also discussed. We thus obtain simple semiphenomenological expressions for the cluster equilibrium distributions and partition functions.
\end{abstract}

\section{Introduction}

The concept of clusters (or droplets) is very useful in a large number of problems in spite of certain ambiguities when referred to realistic (e.g. continuous) systems $s^{1}$ ). In fact non-overlapping clusters can be precisely defined for lattice systems with given interactions between particles; if one then avoids the percolation region in the corresponding phase diagram, the concept of clusters has in principle a practical relevance in phenomena such as nucleation or phase separation ${ }^{2,3}$ ) given that it is then expected to be related to the grains observed by transmission electron microscopy. The situation, however, is not clear cut at present.

It is the purpose of this paper to discuss and try to clarify some ideas concerning the distribution of clusters at equilibrium, in particular the classical nucleation theory and Fisher droplet model ${ }^{4}$ ), the hypothesis about scaling with temperature and magnetic field ${ }^{5,6}$ ) and other recent approaches by Penrose et al.$^{2,3}$ ). We thus find simple expressions for the partition functions and equilibrium distributions of clusters at the coexistence curve and in the one-phase region. This is performed here by comparing theory with data obtained during the computer simulation of the

* Supported in part by NSF Grant DMR81-14726 and by DOE Contract DE-AC02-76 ER03077. 
time evolution of a finite Ising model with Kawasaki dynamics whose details and other results have been described elsewhere ${ }^{7}$ ).

The model used in the simulations consisted of a simple cubic lattice with periodic boundary conditions whose $N(=125000)$ sites are either occupied by a "spin up" or by a "spin down"; alternatively, these two possibilities (which are represented by occupation numbers at each site $i, n_{i}= \pm 1$, respectively) can be interpreted as the site being a particle or an empty site. There is an Ising interaction between nearest-neighbor sites in a way which favors phase segregation, for instance into liquid and vapor phases (in the lattice-gas language). Consequently, the configurational energy of the system is

$$
E=-J \sum_{(i, j)} n_{i} n_{j}, \quad J>0
$$

where the sum goes over all nearest-neighbor pairs of sites.

The dynamics of the system is a Markov process whose basic step is to move one particle to a neighboring empty site with a probability chosen to satisfy detailed balancing,

$$
\exp (-\beta \Delta E) /[1+\exp (-\beta \Delta E)], \quad \beta=1 / k T,
$$

where $k$ is the Boltzmann constant and $\Delta E$ is the increase in energy which would cause the interchange. The number of times this process is attempted, divided by the total number of sites, is taken as the unit of time. This procedure assumes that the system will reach asymptotically a canonical equilibrium state; it also assures that the magnetization $\bar{n}=N^{-1} \Sigma_{i} n_{i}$ and the density $\rho=(1-\bar{n}) / 2, \quad 0 \leqslant \rho \leqslant 1$, $-1 \leqslant \bar{n} \leqslant+1$, will remain constant in time.

The initial state was chosen to be random, corresponding to an infinite temperature. Then the system was quenched to a point in the phase diagram at the temperature $T$ appearing explicitly in the probability (1.2). The phase diagram of the corresponding infinite system is accurately known from series expansions ${ }^{8}$ ), e.g. the critical temperature $T_{\mathrm{c}}$ is very close to $4.51 \mathrm{~J} / \mathrm{K}$. We expect these macroscopic properties to hold approximately for the finite system with $N=125000$, a hope which seems to he confirmed by a comparison of the computed equilibrium energy and magnetic susceptibility with the corresponding known quantities for the infinite Ising $\operatorname{model}^{9}$ ).

The phase points studied in this paper are defined in table I. $P_{1}, P_{2}$ and $P_{3}$ are on the coexistence line at approximately the temperatures $T \approx 0.6 T_{\mathrm{c}}, 0.8 T_{\mathrm{c}}$ and $0.9 T_{\mathrm{c}}$, respectively. $P_{4}(\rho=0.10)$ and $P_{\mathrm{s}}(\rho=0.05)$ are on the one-phase region at $T \approx 0.9 T_{\mathrm{c}} . P_{6}(\rho=0.035)$ is in the one phase region at $T \approx 0.8 T_{\mathrm{c}}$. The line for percolation threshold as a function of the temperature and the coexistence curve are known ${ }^{10}$ ) to intersect at $T_{\mathrm{p}} \approx 0.96 T_{\mathrm{c}}$ (which corresponds to $\rho \approx 0.22$ ); all the above points are thus outside the percolation region. In fact, "infinite" size clusters 
TABLE I

Definition of the phase points considered in this paper. $P_{1}, P_{2}$ and $P_{3}$ are on the coexistence line at different temperatures. $P_{4}$ and $P_{5}$ are on the one-phase region at the same temperature as $P_{3} . P_{6}$ is on the one-phase region at the same temperature as $P_{2} . T, \rho$ and $\bar{n}$ represent the temperature, density and magnetization, respectively. $h$ is the reduced external magnetic field. $\Delta t_{\mathrm{eq}}$ represents the equilibrium time interval during which $m$ different non-correlated measurements were made.

\begin{tabular}{|c|c|c|c|c|c|c|c|}
\hline & $T$ & $\frac{T_{\mathrm{c}}-T}{T_{\mathrm{c}}}$ & $\rho$ & $\bar{n}$ & $h$ & $\begin{array}{c}\Delta t_{\mathrm{eq}} \\
\quad-\end{array}$ & $m$ \\
\hline$P_{1}$ & $8 J / 3 k \approx 0.591 T_{\mathrm{c}}$ & 0.409 & 0.01456 & 0.97088 & 0 & $3000-12785$ & 73 \\
\hline$P_{2}$ & $4 J / 1.137 k \approx 0.780 T_{\mathrm{c}}$ & 0.220 & 0.0613 & 0.8774 & 0 & $2000-4758$ & 70 \\
\hline$P_{3}$ & $4 \mathrm{~J} / \mathrm{k} \approx 0.887 T_{\mathrm{c}}$ & 0.113 & 0.12463 & 0.75074 & 0 & $2000-3420$ & 61 \\
\hline$P_{4}$ & $4 J / k \approx 0.887 T_{\mathrm{c}}$ & 0.113 & 0.10 & 0.80 & 0.024 & $1000-3455$ & 110 \\
\hline$P_{5}$ & $4 J / k \approx 0.887 T_{\mathrm{c}}$ & 0.113 & 0.05 & 0.90 & 0.060 & $1000-4453$ & 116 \\
\hline$P_{6}$ & $4 J / 1.137 k \approx 0.780 T_{c}$ & 0.220 & 0.035 & 0.93 & 0.06 & $1500-4287$ & 94 \\
\hline
\end{tabular}

were never observed during the simulations at $P_{1}-P_{6}$. The system reached equilibrium at those points in a relatively short time (as compared with quenches into the two-phase region or much closer to $T_{\mathrm{c}}{ }^{7}$ ).

As we are only interested in the equilibrium cluster distribution, we first checked that the system had reached the expected equilibrium state by computing specific heats and magnetic susceptibilities $\left.{ }^{9}\right)$. We then let the system to evolve in equilibrium for some time interval $\Delta t_{\mathrm{eq}}$ which is indicated in table I. During this time interval the properties of interest were computed from time to time and then averaged in time. The number of different measurements which were averaged, $m$, is also shown in table I. Typically we let the system to undergo 150000 exchanges between two successive measurements to avoid correlations (this was in fact checked when computing specific heats).

Clusters can be defined unambiguously in our system as the maximal connected set of occupied sites; that is, a cluster is here a set of occupied sites (or "down spins") in the lattice which are mutually connected by at least one nearestneighbor bond. We then investigate the probability $c(l, s)$ that a cluster of "size" $l$ and "energy" $s$ occurs in a unit volume. The size $l$ of a given cluster is defined as the number of particles which belong to it; its energy $s$ is the number of (particle-empty site) bonds (including both, surface and interior bonds) incident on the cluster.

The computer simulations ${ }^{7}$ ) provided data about the cluster size distribution as given by

$$
c_{l}=\sum_{s} c(l, s)
$$

that is, the probability that a cluster of size $l$ occurs in a unit volume. This satisfies 
the sum rule

$$
\rho=\sum_{l} l c_{l} .
$$

They also provided data concerning

$$
s_{l}=\sum_{s} s c(l, s) / \sum_{s} c(l, s),
$$

that is, the mean "energy" corresponding to $l$-size clusters.

\section{Droplet model}

The Fisher droplet model ${ }^{4}$ ) predicts equilibrium clusters at "low" temperature, when they are expected to be compact and independent, distributed in size according to

$$
c_{l}=c_{0} l^{-\tau} \exp \left[-a l^{\sigma}-h l\right],
$$

where $h \equiv \mu H / k T$ is the reduced external magnetic field ( $\mu, H$ and $k$ are, respectively, the magnetic moment per spin, external magnetic field and Boltzmann constant), $c_{0}$ may depend on $T$ and $h$, and $a$ should only depend on the temperature $T$. Different interpretations of eq. (2.1), however, lead to different values for the exponents $\tau$ and $\sigma$.

The original Fisher droplet model assumes $\tau$ independent of temperature and extrapolates the validity of eq. (2.1) to the neighborhood of $T_{\mathrm{c} \text {. }}$ One can then relate $\left.^{4-6}\right) \tau$ and $\sigma$ to the usual exponents $\beta(\approx 5 / 16)$ and $\delta(\approx 5)$ characterizing the critical behavior of the magnetization to obtain

$$
\tau=2+1 / \delta, \quad \sigma=1 / \beta \delta, \quad a=\left(a_{0} J / k T\right) \epsilon,
$$

where $\epsilon \equiv\left(T_{\mathrm{c}}-T\right) / T_{\mathrm{c}}$ and $a_{0}$ is a constant. The exact result $\left.{ }^{11}\right) \ln c_{l} \sim-l^{2 / 3}$ when $l \rightarrow \infty$ suggests to use $\sigma=2 / 3$ in eq. (2.1), instead of $\sigma=0.64$ as implied by relation (2.2). A fit of the resulting equation,

$$
\ln \left(c_{l} l^{\tau}\right)=\ln c_{0}(T)-a(T) l^{2 / 3},
$$

$\tau=2.2$, to the data at the coexistence curve $(h=0)$ gives $a\left(P_{1}\right)=1.756$, $a\left(P_{2}\right)=0.445, a\left(P_{3}\right)=0.087$ and $c_{0}\left(P_{1}\right)=0.217, c_{0}\left(P_{2}\right)=0.159, c_{0}\left(P_{3}\right)=0.144$; this, however, only describes the data for $l>4$ at point $P_{1}, l>14$ at $P_{2}$ and $l>40$ at $P_{3}$. The fit is good at $T=0.59 T_{\mathrm{c}}\left(P_{1}\right)$ but it becomes clearly worse with increasing temperature; in any case it is sensible to the range of $l$ values fitted by the formula. Similar limitations of eq. (2.3) with $\tau=2.2$ were found, for instance, in refs. 2 and 12. 
TABLE II

Values of the parameters in eqs. (2.3) and (2.4) obtained from least-squares fits to the data on different assumptions as explained in the text. Some representative statistical errors bars are also shown.

\begin{tabular}{|c|c|c|c|c|c|c|c|}
\hline \multirow{2}{*}{$\begin{array}{l}\text { Phase } \\
\text { point }\end{array}$} & \multicolumn{3}{|c|}{ Eq. (2.3) } & \multicolumn{2}{|c|}{$\begin{array}{c}\text { Eq. (2.4) } \\
\tau=2.2, \alpha=0.94\end{array}$} & \multicolumn{2}{|c|}{$\begin{array}{c}\text { Eq. (2.4) } \\
\tau=2.09, \alpha=0.88\end{array}$} \\
\hline & $\tau$ & $a$ & $c_{0}$ & $a$ & $c_{0}$ & $a$ & $c_{0}$ \\
\hline$P_{1}$ & $0.90 \pm 0.03$ & $2.21 \pm 0.02$ & 0.091 & $1.40 \pm 0.02$ & 0.058 & 1.40 & 0.055 \\
\hline$P_{2}$ & $1.40 \pm 0.03$ & $0.57 \pm 0.02$ & 0.036 & $0.36 \pm 0.02$ & 0.096 & 0.38 & 0.082 \\
\hline$P_{3}$ & $1.65 \pm 0.03$ & $0.12 \pm 0.02$ & 0.023 & $0.088 \pm 0.002$ & 0.177 & 0.098 & 0.123 \\
\hline
\end{tabular}

One may then be tempted to drop the term $l^{\tau}$ in eq. (2.3); this (or some other non-dominant $l$-correction) seems, however, important for the range of temperatures and $l$-values considered here. In fact, putting $\tau=0$ in eq. (2.3) makes $a(T)$ to change monotonicaly from 2.7 (when one fits $2 \leqslant l \leqslant 10$ ) to 2.5 $(7 \leqslant l \leqslant 10)$ at $0.59 T_{\mathrm{c}}$, from $0.88(5 \leqslant l \leqslant 45)$ to $0.79(20 \leqslant l \leqslant 45)$ at $0.78 T_{\mathrm{c}}$, and from $0.28(10 \leqslant l \leqslant 200)$ to $0.22(80 \leqslant l \leqslant 200)$ at $0.89 T_{\mathrm{c}}$.

Allowing for a $T$-dependence on $\tau$ in eq. (2.3) leads to a much better description of the data than any of the two previous choices. A least-squares fit and the use of the sum rule (1.4) for the points at the coexistence curve gives the parameters in table II. The overall differences between the formula and the data are smaller than $1.5 \%$ so that the description is indeed very satisfactory. A closer look, however, shows that the formula systematically tends to underestimate the probability of a monomer $(l=1)$ (up to $14 \%$ at $0.89 T_{\mathrm{c}}$ ) while it overestimates that of clusters with small $l>1$. Thus we have also tried to exclude large clusters from the fit with the result of an increase of the overall differences to $4 \%$ because the formula then cannot extrapolate so well to large $l$ values. While those are probably acceptable discrepancies, one may find some good reasons to look for a different approach. Namely that eq. (2.3) contains as much as three temperature-dependent parameters (where $\tau$ should perhaps represent only a geometrical effect, independent of $T$ ) and that it cannot be extrapolated near $T_{\mathrm{c}}$ because it then violates scaling relations such as (2.2).

As an alternative to other proposals ${ }^{13}$ ) designed for specific purposes ${ }^{14}$ ), we find some evidence that a formula such as

$$
c_{l}=c_{0} l^{-\tau} \exp \left(-a l^{2 / 3}\right)\left[1-\alpha \exp \left(-a l^{2 / 3}\right)\right],
$$

where $a=a(T)$ while $\tau$ and $\alpha$ are independent of temperature, may be a good representation of independent clusters along the coexistence curve $(h=0)$ up to $T_{\mathrm{c}}$. Note, however, that our definition of clusters in section 1 would not be appropriate to check the validity of eq. (2.4) from the vicinity of the percolation threshold $T_{\mathrm{p}}=0.96 T_{\mathrm{c}}$ up to $T_{\mathrm{c}}$. 


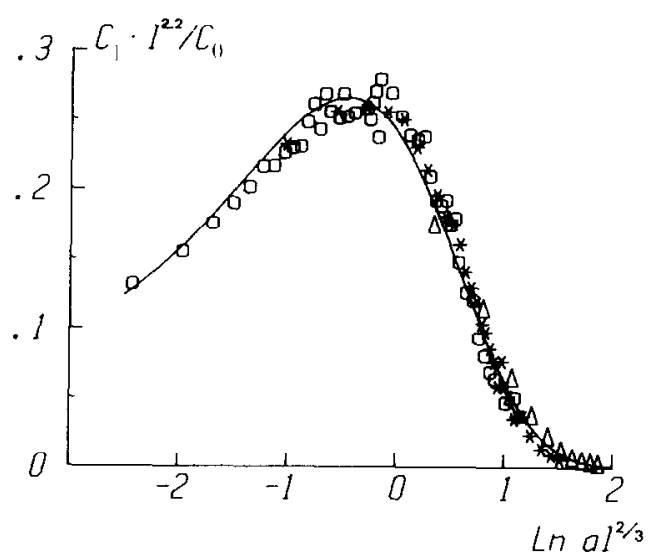

Fig. 1. The data at $P_{1}$ (triangles) $P_{2}$ (asterisks) and $P_{3}$ (circles) (coexistence curve) is compared with eq. (2.4) when $\tau=2.2, \alpha=0.94$ and $a(T)$ given in table II (solid line). The parameter $c_{0}(T)$ was computed using the sum rule (1.4). A similar plot using eq. (2.3) with a temperature-dependent exponent $\tau$, as in table II, produces more dispersion of the data points and disagreements with the theoretical line at high temperatures (see the text).

The Fisher's factor in eq. (2.4) states the probability of a cluster while the term in square brackets would then represent an approximation to the probability of empty sites surrounding that cluster. We have assumed here $\tau=2.2$ (see, however, section 6). Requiring a common value for $\alpha$, the best fit to the data is given at different temperatures by the values of $a(T)$ in table II where $c_{0}$ follows from the use of the sum rule (1.4); the overall differences between theory and experiment are then $1.8,3,5 \%$, respectively, at $T / T_{\mathrm{c}}=0.89,0.78$ and 0.59 , i.e. they decrease with increasing temperature. These differences can be reduced by a factor of $1 / 2$ by excluding large $l$ values (where the data statistics is worse) from the leastsquares fit. Thus, eq. (2.4) with $\tau$ and $\alpha$ independent of $T$ seems preferable, even numerically, to eq. (2.3) with a $T$-dependent exponent $\tau$; this fact is particularly clear at $T \geqslant 0.78 T_{\mathrm{c}}$ (see also section 3 ). We have combined in fig. 1 the data at the three phase points for $h=0$ by using in eq. (2.4), $\tau=2.2$, the parameters in table II.

\section{Very low temperatures}

Lebowitz and Penrose ${ }^{15}$ ) have shown that one can state bounds for $c_{l}$,

$$
(1-\rho)^{2+5 l} \leqslant c_{l} / Q_{l} z^{l} \leqslant(1+z)^{-b(l)-l},
$$

where $b(l)$ is the minimum perimeter for $l$-particle clusters, $2+5 l=l+$ maximum perimeter for an $l$-cluster, $z=\exp (-12 J / k T)$ is the system fugacity along the 
TABLE III

Values of the parameters in eq. (2.3) obtained from a least-squares fit to the bounds (3.1) for $T<0.5 T_{\mathrm{v}}$

\begin{tabular}{lclll}
\hline & \multicolumn{3}{c}{ Eq. (2.3) } & \multirow{2}{*}{ Eq. (2.3), $\tau=0$} \\
\cline { 2 - 4 }$T / T_{\mathrm{c}}$ & $\tau$ & $a$ & error & $a$ \\
\hline 0.493 & 0.64 & 3.54 & $0.5 \%$ & 4.0 \\
0.443 & - & - & - & 4.8 \\
0.403 & 0.06 & 5.57 & $0.7 \%$ & - \\
0.386 & -0.07 & 6.06 & $0.8 \%$ & - \\
0.355 & - & - & - & 6.8 \\
0.296 & -0.78 & 9.33 & $1.8 \%$ & - \\
0.177 & - & - & - & 16.1 \\
\hline
\end{tabular}

coexistence line, and $Q_{l}$ is the "cluster partition function". The latter is defined as

$$
Q_{l}=\sum_{k}^{\prime} \mathrm{e}^{-E(K) / k T},
$$

where $E(K)=-4 J n(K)$ is the (negative) energy of the cluster $K$, with $n(K)$ the number of adjacent pairs of occupied sites on that cluster, and the sum includes one member from each set of translationally non-equivalent $l$-clusters. Thus $Q_{l}$ are polynomials in $\exp (4 J / k T) ; Q_{1} \equiv 1$. The coefficients for these polynomials were computed exactly by Sykes ${ }^{16}$ ) from $l=1$ to $l=10$ for the simple cubic lattice $\left(4 J / k T \approx 0.88669 T_{\mathrm{c}} / T\right)$.

The bounds (3.1) at a given temperature become indistinguishable when $T \leqslant 0.5 T_{\text {c }}$; they can then be used to check the alternatives described in section 2 .

A fit of the bounds (3.1) to eq. (2.3) when one allows a $T$-dependence on $\tau$ or uses $\tau=0$ gives the values quoted in table III. This clearly shows that the description provided by the classical nucleation theory does not become better with decreasing temperature. When fitting $1 \leqslant l \leqslant 6\left(c_{l}\right.$ is negligible for $\left.l>6\right)$ we find that the numerical bounds (3.1) imply a clear curvature on a $\ln c_{l}$ versus $l^{2 / 3}$ plot, the curvature showing a change of sign (i.e., $\tau=0$ ) when $T \approx 0.39 T_{\mathrm{c}}$. The overall differences between the theory and the data systematically increase with decreasing temperature. Our modification (2.4) does not make a better job at such low temperatures (where it is not intended to work).

\section{Small clusters}

Let us refer now to a different approach ${ }^{2}$ ) to the equilibrium cluster distribution which was shown to be useful when analysing kinetic phenomena ${ }^{2,3}$ ). 
The bounds (3.1) are not close enough to give accurate information at $\rho>0.02$, namely for $T \geqslant 0.6 T_{\mathrm{c}}$ along the coexistence curve. They, however, suggest to look for expressions

$$
c_{l} \approx Q_{1} w^{l}(1-\rho)^{k_{l}},
$$

where $w=w(T, \rho)$ is a renormalized "fugacity".

In order to check the validity of eq. (4.1) we have computed the ratios $c_{l+1} Q_{l} / c_{l} Q_{l+1}$ taking our data at points $P_{1}-P_{6}$ for $c_{l}$ and Sykes' exact results ${ }^{16}$ ) for $Q_{l}$. These ratios become constant for $l \geqslant 3$ implying that $k_{l}$ in eq. (4.1) is also constant for $l \geqslant 3$. By computing

$$
k_{l} \equiv \frac{\ln \left(c_{l} / \bar{w}^{l} Q_{l}\right)}{\ln (l-\rho)}, \quad \bar{w} \equiv \frac{1}{7} \sum_{l-3}^{9} \frac{c_{l+1} Q_{l}}{c_{l} Q_{l+1}},
$$

we then find

$$
k_{1}=3.25, \quad k_{2}=4.5 ; \quad k_{l} \approx 5, \quad l \geqslant 3 .
$$

Interesting enough the exponents (4.3) (and eqs. (4.1)) are formally consistent with the exact values for $c_{l}$ at infinite temperature. At $T=\infty$, when the occupation variables $n_{i}$ in the Hamiltonian (1.1) are independent, one clearly has ${ }^{15}$ )

$$
c_{l}=\sum_{K}^{\prime} \rho^{n(K)}(1-\rho)^{n^{\prime}(K)},
$$

where $n(K)=l$ and $n^{\prime}(K)$ is the number of sites not in $K$ that are neighbors of sites in $K$. Combining eqs: (4.1) and (4.4) for $l \leqslant 4$ we obtain

$$
\begin{aligned}
& 2 k_{1}-k_{2}=2, \quad 86(1-\rho)^{k_{4}}=\left(86-108 \rho+57 \rho^{2}-3 \rho^{3}\right)(1-\rho)^{4 k_{1}-9}, \\
& 5(1-\rho)^{k_{3}}=(5-\rho)(1-\rho)^{3 k_{1}-5}, \quad w=\rho(1-\rho)^{6-k_{1}}
\end{aligned}
$$

at $T=\infty$; these relations are fully consistent with (4.3).

The renormalized fugacity $w$ in eq. (4.1) can be evaluated by computing

$$
\rho_{10} \equiv \sum_{l=1}^{10} l c_{l}, \quad \epsilon_{10} \equiv \sum_{l=1}^{10} s_{l} c_{l}
$$

from our data at points $P_{1}-P_{6}$ and realizing that eq. (4.1) implies

$$
\rho_{10}=\sum_{l=1}^{10} l w^{l}(1-\rho)^{k_{1}} Q_{1}, \quad \epsilon_{10}=\sum_{l=1}^{10} s_{l} w^{l}(1-\rho)^{k_{l}} Q_{l} .
$$

Here we know the exact values for $Q_{l}$ and we found independently that

$$
s_{l}=\sum_{(n)}(6 l-2 n) Q(n) \mathrm{e}^{4 J n / k T} / \sum_{(n)} Q_{N}(n) \mathrm{e}^{4 J n / k T},
$$


where the sums go only over the $n$-values appearing as exponents at each polynomial $Q_{l}$ (see section 3 ) and $(6 l-2 n)$ is the number of bonds within a cluster. Using Newton's method we obtain, for given $T$ and $h$, the same value for $w$ (to a very good approximation) from any of the two eqs. (4.7) with exponents (4.3). The resulting $w=w(T, h)$, together with our experimental values for $\rho_{10}$ and $\epsilon_{10}$, are given in table IV. The predictions of eq. (4.1) for $l \leqslant 10$ are then compared in tables $\mathrm{V}-\mathrm{X}$ with the computer data; the agreement is very good.

\section{TABLE IV}

Values for $\rho_{10}$ and $\epsilon_{10}$ as defined in eqs. (4.6) obtained from the computer simulation data, and values for the renormalized fugacity $w$ as computed from eqs. (4.7). The shown fugacity $w_{5}$ at $P_{1}$, however, was computed from $c_{i}, l<7$.

\begin{tabular}{llll}
\hline & $\rho_{\text {10 }}$ & $\epsilon_{10}$ & $w$ \\
\hline$P_{1}$ & 0.01455 & 0.08203 & 0.010560 \\
$P_{2}$ & 0.05614 & 0.28808 & 0.027481 \\
$P_{3}$ & 0.07196 & 0.35802 & 0.036050 \\
$P_{4}$ & 0.07460 & 0.37311 & 0.035190 \\
$P_{5}$ & 0.04920 & 0.26095 & 0.028015 \\
$P_{6}$ & 0.03484 & 0.18880 & 0.021477 \\
\hline
\end{tabular}

TABLE V

The computer data for $c_{l}$ at $P_{1}$ (coexistence curve) is compared with the corresponding values predicted by eq. (4.1) with the parameters (4.3) and $w$ given in table IV. The values in brackets correspond to a $168 \times 168 \times 168$ system with Glauber dynamics and were kindly provided to us by D. Stauffer and D.W. Heermann, see ref. 20. (Our values for $l \geqslant 7$ present large statistical errors so that they were not included in the computations.) The overall differences between theory and experiment are $1.5 \%$.

\begin{tabular}{rrr}
\hline & \multicolumn{2}{c}{$T=0.59 T_{\mathrm{c}}, \rho=0.01456$} \\
$50^{3} \times c_{l}$ \\
$l$ & experiment & theory \\
\hline 1 & $1256.55(1260.35)$ & 1258.55 \\
2 & $175.42(175.62)$ & 175.44 \\
3 & $41.59(40.73)$ & 41.21 \\
4 & $12.66(12.35)$ & 12.54 \\
5 & $4.29(4.36)$ & 4.31 \\
6 & $1.55(1.57)$ & 1.62 \\
7 & $-(0.569)$ & 0.643 \\
8 & $-(0.259)$ & 0.267 \\
9 & $-(0.123)$ & 0.115 \\
10 & $-(0.048)$ & 0.051 \\
\hline
\end{tabular}


TABLE VI

The computer data at $P_{2}$ (coexistence curve) is compared with eq. (4.1) (with the parameters (4.3) and $w$ given in table IV) when $l \leqslant 10$ and with eq. (5.3) and (5.2) with $\tau=2.09, \alpha=0.88$ and $a$ given in table II when $l>10$. The agreement is very good up to $l \approx 20$; it is also reasonable for $l>20$ where the experimental data may suffer from large statistical errors and finite size effects. The overall $(1 \leqslant l \leqslant 45)$ differences between theory and experiment are $2.2 \%$.

\begin{tabular}{rrr}
\hline & \multicolumn{2}{c}{$T=0.78 T_{c}, \rho=0.0613$} \\
& \multicolumn{2}{c}{$50^{3} \times c_{1}$} \\
\hline & experiment & theory \\
\hline & 2781.60 & 2796.76 \\
1 & 668.79 & 664.14 \\
3 & 277.03 & 275.63 \\
4 & 145.11 & 145.38 \\
5 & 87.03 & 85.72 \\
6 & 53.59 & 54.16 \\
7 & 35.50 & 35.91 \\
8 & 24.23 & 24.66 \\
9 & 17.24 & 17.39 \\
10 & 13.23 & 12.52 \\
11 & 9.84 & 9.32 \\
12 & 7.13 & 7.06 \\
13 & 5.50 & 5.43 \\
14 & 4.30 & 4.23 \\
15 & 3.21 & 3.34 \\
16 & 2.63 & 2.65 \\
18 & 1.57 & 1.73 \\
20 & 1.25 & 1.16 \\
23 & 0.557 & 0.670 \\
27 & 0.314 & 0.343 \\
30 & 0.157 & 0.216 \\
35 & 0.063 & 0.106 \\
40 & 0.030 & 0.055 \\
45 & 0.017 & 0.030 \\
\hline
\end{tabular}

The values (4.3) differ from those in ref. $2\left(k_{1}=3, k_{l}=4, l \geqslant 2\right)$ where the analysis was namely focused on $T=0.59 T_{\mathrm{c}}$ and based on much more limited data. These values for $k_{l}$ produce overall differences between experimental and theoretical $c_{i}$ 's larger than $1.5 \%$ while in the case of (4.3) these are smaller than $0.7 \%$. This preference of the data for (4.3) becomes much more evident for $l>10$, specially at high temperatures, say $T=0.89 T_{\mathrm{c}}$. 
TABLE VII

Same as table VI at $\boldsymbol{P}_{3}$ (coexistence curve). The overall differences are smaller than $1.5 \%$.

\begin{tabular}{|c|c|c|}
\hline \multirow[b]{2}{*}{$l$} & \multicolumn{2}{|c|}{$\begin{array}{c}T=0.887 T_{\mathrm{c}}, \rho=0.12463 \\
50^{3} \times c_{l}\end{array}$} \\
\hline & experiment & theory \\
\hline 1 & 2908.90 & 2923.71 \\
\hline 2 & 744.95 & 727.77 \\
\hline 3 & 345.84 & 333.63 \\
\hline 4 & 198.92 & 198.68 \\
\hline 5 & 129.16 & 131.67 \\
\hline 6 & 92.79 & 93.10 \\
\hline 7 & 66.20 & 68.86 \\
\hline 8 & 51.53 & 52.62 \\
\hline 9 & 40.38 & 41.22 \\
\hline 10 & 32.16 & 32.92 \\
\hline 13 & 19.03 & 19.28 \\
\hline 16 & 12.59 & 12.47 \\
\hline 19 & 8.57 & 8.60 \\
\hline 21 & 6.95 & 6.89 \\
\hline 25 & 4.78 & 4.64 \\
\hline 30 & 3.47 & 3.03 \\
\hline 40 & 1.67 & 1.49 \\
\hline 50 & 0.953 & 0.826 \\
\hline 60 & 0.567 & 0.498 \\
\hline 70 & 0.364 & 0.318 \\
\hline 80 & 0.251 & 0.212 \\
\hline 90 & 0.164 & 0.146 \\
\hline 100 & 0.111 & 0.103 \\
\hline 110 & 0.086 & 0.075 \\
\hline 120 & 0.055 & 0.055 \\
\hline 130 & 0.040 & 0.041 \\
\hline 140 & 0.029 & 0.032 \\
\hline 150 & 0.023 & 0.024 \\
\hline 175 & 0.012 & 0.013 \\
\hline 200 & 0.010 & 0.008 \\
\hline
\end{tabular}

\section{Partition functions for large clusters}

We now extrapolate the $Q_{l}$ partition functions, which are only known exactly up to $l=10$, to larger sizes, $l>10$. Let $w_{l} \equiv Q_{l} / Q_{l+1}$; it then follows from eq. (4.1) with (4.3) when $l \geqslant 3$ :

$$
w_{l}=w \frac{c_{l}}{c_{l+1}} \approx w \exp \left[-\left(\frac{\partial \ln c_{l}}{\partial l}\right)_{l+1 / 2}\right] \text {. }
$$


TABLE VIII

Same as table VI at $P_{4}$ (one-phase region). The overall differences are $2.2 \%$.

\begin{tabular}{rrr}
\hline & \multicolumn{2}{c}{$T=0.887 T_{\mathrm{c}}, \rho=0.10$} \\
& \multicolumn{2}{c}{$50^{3} \times c_{l}$} \\
\cline { 2 - 3 }$l$ & experiment & theory \\
\hline 1 & 3110.57 & 3123.33 \\
2 & 799.35 & 785.70 \\
3 & 365.84 & 356.50 \\
4 & 206.25 & 207.23 \\
5 & 131.32 & 134.06 \\
6 & 89.34 & 92.53 \\
7 & 66.42 & 66.81 \\
8 & 49.16 & 49.84 \\
9 & 38.22 & 38.11 \\
10 & 29.90 & 29.71 \\
13 & 16.14 & 16.19 \\
15 & 11.42 & 11.43 \\
18 & 7.05 & 7.20 \\
21 & 4.83 & 4.77 \\
25 & 3.00 & 2.92 \\
29 & 1.89 & 1.87 \\
35 & 1.01 & 1.02 \\
40 & 0.673 & 0.649 \\
50 & 0.258 & 0.284 \\
60 & 0.108 & 0.134 \\
70 & 0.046 & 0.067 \\
80 & 0.023 & 0.035 \\
90 & 0.011 & 0.019 \\
100 & 0.007 & 0.011 \\
\hline & & \\
\hline 0 & &
\end{tabular}

Inserting here expression (2.4) for $c_{l}$ and writing $w_{\mathrm{s}} \equiv w(T, h=0)$ we have

$$
w_{l}=w_{\mathrm{s}} \exp \left\{\frac{\tau}{l+1 / 2}+\frac{2}{3} a\left(l+\frac{1}{2}\right)^{-1 / 3}\left[1-\frac{1}{\alpha^{-1} \exp \left[a(l+1 / 2)^{2 / 3}\right]-1}\right]\right\} .
$$

One also has from eq. (4.1) that

$$
c_{l}=c_{l_{0}} w^{l-l_{0}} \prod_{l=l_{0}}^{l-1} w_{l}^{-1}, \quad l_{0} \geqslant 3 .
$$

This allows to compute $c_{l}$ from a given $c_{l_{0}}$ at any temperature and magnetic field when one uses here the corresponding value for $w$ (as given in table IV) and the expression (5.2) with the appropriate values for the parameters $\tau$ and $a$. Using $\tau=2.2$ and $a(T)$ as given in table II we find a very good agreement with the computer data at points $P_{1}-P_{6}$ (see section 6). 
TABLE IX

Same as table VI at $\boldsymbol{P}_{5}$ (one-phase region). The overall differences are smaller than $1.8 \%$.

\begin{tabular}{|c|c|c|}
\hline \multirow[b]{2}{*}{$l$} & \multicolumn{2}{|c|}{$\begin{array}{c}T=0.887 T_{\mathfrak{c}}, \rho=0.05 \\
50^{3} \times c_{l}\end{array}$} \\
\hline & experiment & theory \\
\hline 1 & 2959.22 & 2964.16 \\
\hline 2 & 639.47 & 635.13 \\
\hline 3 & 237.84 & 235.71 \\
\hline 4 & 107.05 & 109.08 \\
\hline 5 & 55.82 & 56.18 \\
\hline 6 & 30.57 & 30.87 \\
\hline 7 & 17.31 & 17.74 \\
\hline 8 & 10.79 & 10.54 \\
\hline 9 & 6.56 & 6.41 \\
\hline 10 & 4.16 & 3.98 \\
\hline 12 & 1.59 & 1.66 \\
\hline 14 & 0.73 & 0.73 \\
\hline 16 & 0.285 & 0.332 \\
\hline 18 & 0.155 & 0.156 \\
\hline 20 & 0.069 & 0.075 \\
\hline 25 & 0.014 & 0.013 \\
\hline
\end{tabular}

TABle $X$

Same as table VI at $\boldsymbol{P}_{6}$ (one-phase region). The overall differences are $5 \%$.

\begin{tabular}{rrr}
\hline & \multicolumn{2}{c}{$T=0.78 T_{\mathrm{c}} \rho=0.035$} \\
& \multicolumn{2}{c}{$50^{3} \times c_{i}$} \\
\cline { 2 - 3 }$l$ & \multicolumn{1}{c}{ experimental } & theory \\
\cline { 2 - 3 } 1 & 2389.27 & 2391.10 \\
2 & 460.15 & 459.35 \\
3 & 151.88 & 151.06 \\
4 & 62.41 & 62.27 \\
5 & 28.84 & 28.69 \\
6 & 13.61 & 14.17 \\
7 & 7.48 & 7.34 \\
8 & 3.75 & 3.94 \\
9 & 2.20 & 2.17 \\
10 & 1.19 & 1.22 \\
12 & 0.447 & 0.421 \\
14 & 0.149 & 0.154 \\
20 & 0.017 & 0.010 \\
\hline
\end{tabular}


Dropping the term in square brackets in eq. (5.2) is equivalent to use eq. (2.3) instead of (2.4) for $c_{l}$ in eq. (5.1). The resulting equation for $w_{l}$ can then be used in eq. (5.3) together with the parameters $\tau(T)$ and $a(T)$ in table II. The agreement with the computer data at points $P_{1}-P_{6}$ is then reasonably good but the overall differences are twice the ones when using eq. (5.2) which only contains a temperature dependent parameter, $a(T)$ (see following section for numerical and graphical evidences).

\section{Scaling behavior}

The relevance of the modified droplet formula (2.4), which allows to keep the exponent $\tau$ independent of temperature (unlike the situation when one considers eq. (2.3)), thus decreasing the number of free parameters, shows up again when considering scaling ideas.

The homogeneity of the magnetization implies, given the sum rule (1.4), a similar property for $c_{l}$. Moreover, one expects that both the energy $s$ and the size $l$ will be needed to describe the loose clusters occurring near (and not so near) $T_{\mathrm{c}}$. Thus, homogeneity and the need for a new exponent related to $s$ lead Binder to assume ${ }^{5.6}$ ) that near $T_{\mathrm{c}}$ and for small magnetic fields ${ }^{17}$ ), one has

$$
c(l, s)=l^{-\tau_{0}} f_{1}\left(\epsilon l^{z}, h l^{v}, s l^{-r}\right) \text {. }
$$

where the exponents $\tau_{0}, x, y$ and $z$ are to be determined. This can be accomplished in part by combining eqs. (6.1), (1.4) and (1.1) to write

$$
E=-J N\left[3-\sum_{s . l} s c(l, s)\right] \text {, }
$$

from where it follows

$$
\tau_{0}=2+x+y / \delta, \quad z=y / \beta \delta, \quad x=1-y+y / \beta \delta .
$$

Thus only one of the exponents in eq. (2.1), e.g. $y$, is left undetermined.

Summing eq. (6.1) over $s$ one is led to

$$
c_{l}=l^{-\tau} f_{2}\left(\epsilon l^{y / \beta \delta}, h l^{y}\right), \quad \tau=\tau_{0}-x=2+y / \delta,
$$

according to the definition (1.3). The scaling form (6.4) can be checked against our data at points $P_{1}-P_{3}(h=0)$ by plotting $c_{i} l^{2+y / \delta}$ versus $\epsilon l^{y / \beta \delta}$. It turns out that this plot is very sensitive to the value of the exponent $y$ and that one indeed obtains a unique curve, independent of temperature for points $P_{2}$ and $P_{3}$, only for a very narrow range of $y$ values; the best fit is given by

$$
y=0.45 \pm 0.02 .
$$




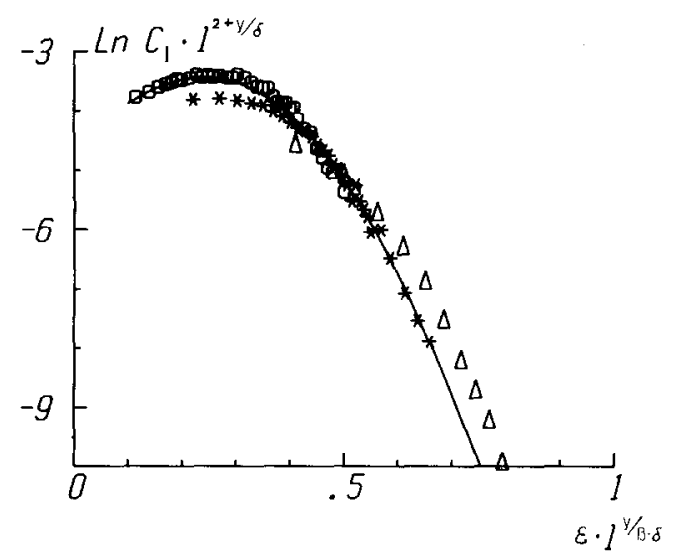

Fig. 2. Evidence for scaling behavior of the cluster distribution with temperature. The circles are for $P_{3}\left(0.89 T_{\mathrm{c}}\right)$ and the asterisks for $P_{2}\left(0.78 T_{\mathrm{c}}\right)$, both on the coexistence line. The solid line is a polynomic fit to the data: $-4.608+8.634 v+9.534 v^{2}-29.346 v^{3}+18.816 v^{4}$ with $v \equiv \epsilon l^{y / \beta \delta}$. The triangles (for $P_{1}, 0.59 T_{\mathrm{c}}$ ) also lie close to this fit. Here $\beta=5 / 16, \delta=5$ and $y=0.45$.

Even the data at $P_{1}$ lies close to the other. The evidence for (6.4) and (6.5) is given in fig. 2 where we included a polynomic fit to the data. The result (6.5) is to be compared with the value $y=1$ implied by the Fisher droplet model (section 2) and with the value $y=0.5$ guessed by Müller-Krumbhaar and Stoll ${ }^{6}$ ).

In order to check the scaling of our data with an external magnetic field (and get a stringent test of eqs. (6.4) and (6.5)) we have to assume factorization of the function $f_{2}$ in eq. (6.4),

$$
c_{l} l^{2+y / \delta}=f_{3}\left(\epsilon l^{y / \beta \delta}\right) f_{4}\left(h l^{y}\right),
$$

a fact which is included in most approaches. Thus we expect, writing $c_{l}^{\mathrm{s}} \equiv c_{l}(h=0)$,

$$
c_{l} / c_{l}^{\mathrm{s}}=f_{5}\left(h l^{y}\right) \text {, }
$$

where $f_{5}(m)=f_{4}(m) / f_{4}(0)$, which is confirmed very well in fig. 3 by our data at points $P_{4}, P_{5}$ and $P_{6}$ when $y=0.45$ and $h_{\mathrm{r}}\left(P_{4}\right) \equiv 1, h_{\mathrm{r}}\left(P_{5}\right)=2.41$ and $h_{\mathrm{r}}\left(P_{6}\right)=2.56$. These relative values for the field were obtained approximately assuming at point $P_{4}$ that $\delta H \approx 2 \mu N \delta \rho / \chi_{\mathrm{T}}$, where $\chi_{\mathrm{T}}$ is the magnetic susceptibility, using at $P_{5}$ the known relations ${ }^{18}$ ) between the asymptotic equations of state for the sc and bcc Ising model, and using mean field relations to compare $P_{6}$ with $P_{4}$ and $P_{5}$. In any case the uncertainty in our values for $h(T, \rho)$ does not seem to affect dramatically the above results.

Of course, $0.59 T_{\mathrm{c}}$ is far away from $T_{\mathrm{c}}$ to be considered when checking scaling ideas so that one should not overestimate the fact that $P_{1}$ has a qualitative 


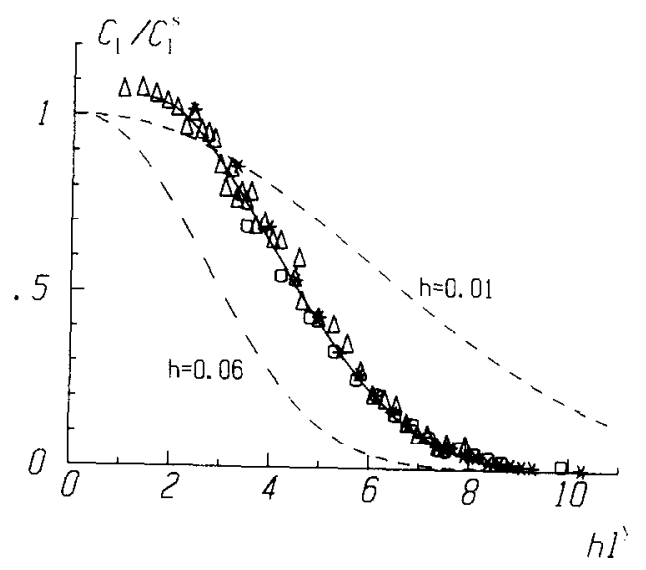

Fig. 3. Evidence for scaling behavior of the cluster distribution with magnetic field. The triangles are for $P_{4}\left(0.89 T_{c}, \rho=0.10\right)$, the asterisks for $P_{6}\left(0.89 T_{c}, \rho=0.05\right)$ and the circles for $P_{6}\left(0.78 T_{c}\right.$, $\rho=0.035)$. Here $y=0.45, h_{\mathrm{r}}\left(P_{4}\right) \equiv 1, h_{\mathrm{r}}\left(P_{5}\right)=2.41$ and $h_{\mathrm{r}}\left(P_{\mathrm{b}}\right)=2.56$. The solid line is a polynomic fit to the data: $0.767+0.440 v-0.201 v^{2}+2.46 \times 10^{-2} v^{3}-9.73 \times 10^{-4} v^{4}$ with $v \equiv h_{\mathrm{r}} l^{y}$. The dashed lines correspond to the function $\mathrm{e}^{-h l}$ for small values of $h$, a behavior implied by the classical exponent $y=1$.

behavior close to the one at $P_{3}$. Equations such as $(6.1),(6.4),(6.6)$ or $(6.7)$ are only valid asymptotically, when $T \rightarrow T_{c}$, and we observe systematic (although small) deviations of the data at point $P_{1}$ from scaling (see, for instance, fig. 2 ). Further evidences about scaling can be found in ref. 23 .

Interesting enough, the modification (2.4) of the classical droplet model is consistent with the above scaling hypothesis. If scaling is granted and one writes consequently

$$
a=a_{0} \epsilon^{t},
$$

the validity of eq. (2.4) near $T_{\mathrm{c}}$ implies (note that we are assuming $\sigma=2 / 3$ from the beginning)

$$
\begin{aligned}
& \tau=2+y / \delta=2.09 \pm 0.01, \\
& t=\beta \delta \sigma / y=2.31 \pm 0.10
\end{aligned}
$$

after using (6.5).

The sum-rule (1.4) can thus be used to determine the parameter $c_{0}$ and we are only left with the constants $a_{0}$ in eq. (6.8) and $\alpha$ in eq. (2.4), both independent of temperature, to adjust the data. In practice we have adjusted eq. (2.4) to our data to obtain $a(T)$ (instead of $a_{0}$ ) and $\alpha$ (independent of $T$ ); $c_{0}$ follows then from eq. (1.4). We thus obtain the values reported in table II which are not very different 


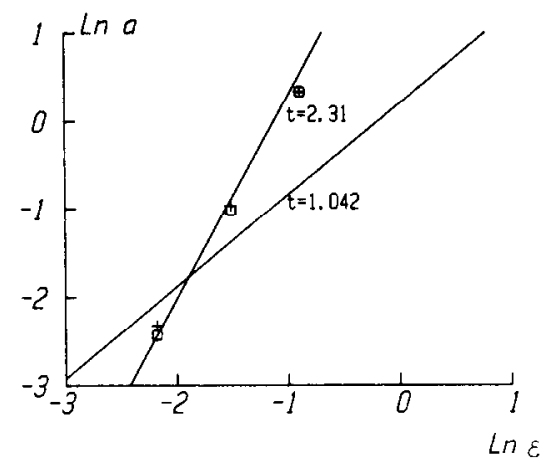

Fig. 4. Log-log plot of the values of the parameter $a(T)$ in table II versus $\epsilon=\left(T_{\mathrm{c}}-T\right) / T_{\mathrm{c}}$ to show that the values when $\tau=2.09$ (crosses) are consistent with the relation $a=a_{0} \epsilon^{2.31}$ while the values when $\tau=2.2$ (circles) do not seem consistent with $a=a_{0} \epsilon^{1.042}$ (see eq. (6.8)).

from the ones obtained in section 2 (also reported in table II) when $\tau=2.2$; the new values, however, provide an even better fit and present the advantage of making eq. (2.4) valid near $T_{\mathrm{c}}$ as well as in the range considered here, $0.6 \leqslant T / T_{\mathrm{c}} \leqslant 0.9$. Fig. 4 evidences that the values $a(T)$ obtained from this fit are fully consistent with the behavior (6.8) with $t=2.31$, while the values for $a(T)$ in table II when $\tau=2.2$ are not consistent with the value $t=1.042$ following from eq. (6.9) in the "classical" case $y=1$,

The evidence that eq. (2.4) with $\tau=2.09$ is a very good description of the data over the range $0.6<T / T_{\mathrm{c}} \leqslant 0.9$ (one perhaps observes systematic discrepancies at $0.59 T_{\mathrm{c}}$ ) is given in fig. 5 where we present a plot $\ln c_{l}$ versus $l^{2 / 3}$ of the data at points $P_{1}-P_{3}$ together with the corresponding theoretical lines.

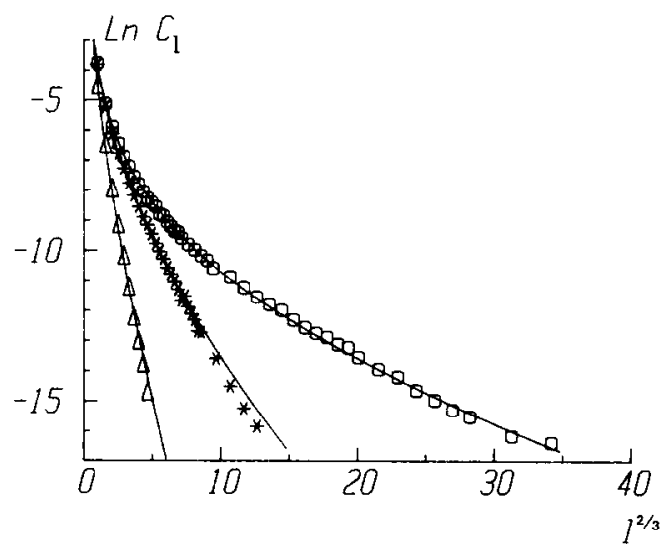

Fig. 5. Plot of $\ln c_{i}$ versus $l^{2 / 3}$ to compare the data at $P_{1}$ (triangles). $P_{2}$ (asterisks) and $P_{3}$ (circles) ( $h=0$ ) with eq. (2.4) when $\tau=2.09, \alpha=0.88$ and $a(T)$ as given in table II (solid lines). The parameter $c_{0}$ was computed using the sum rule (1.4). The agreement is slightly better than in fig. 1 where $\tau=2.2$ was assumed. 
The resulting eq. (2.4) with $\tau=2.09$ can also be incorporated into the approach described in section 5; it then follows eq. (5.2) with $\tau=2.09$ and $a(T)$ as given in table II. We present in tables $\mathrm{V}-\mathrm{X}$ a comparison between the experimental cluster distributions at points $P_{1}-P_{6}$ and the predictions of eq. (5.3), $l>10$, with $c_{l_{0}}=c_{10}=Q_{10} w^{10}(1-\rho)^{5}$ and $w$ given by table IV. The agreement is certainly good, a fact which stresses the relevance of eq. (2.4): note that we use the same values for the parameters $\tau, \alpha$ and $a(T)$ when describing the data with eqs. (5.2) and (5.3) than when they are described directly by eq. (2.4).

The present approach takes care of the case $h \neq 0$ by using $w$ (instead of $w_{\mathrm{s}}$ at the coexistence curve, $h=0$ ) in eqs. (4.1) or (5.3). We thus have,

$$
c_{l}=c_{l}^{\mathrm{s}}\left(\frac{w}{w_{\mathrm{s}}}\right)^{l}\left[\frac{1-\rho}{1-\rho_{\mathrm{s}}}\right]^{k_{l}}
$$

$k_{l}=5$ for $l \geqslant 3, c_{l}^{\mathrm{s}} \equiv c(h=0)$. It follows that

$$
\ln \tilde{c}_{l} \equiv \ln \left\{c_{l}\left(\frac{w_{\mathrm{s}}}{w}\right)^{\prime}\left[\frac{1-\rho_{\mathrm{s}}}{1-\rho}\right]^{k_{l}}\right\}
$$

should give the same function as $\ln c_{l}^{s}$; the evidence for this fact is given in fig. 6 .

The relation (6.10), valid for any value of the field $h$, should be consistent with the scaling relation (6.7) when $h$ is small enough. Assuming $f_{5}(\xi)=\exp \left(\right.$ const. $\xi^{1 / y}$ ) in eq. (6.7) we have

$$
\ln \left(w / w_{\mathrm{s}}\right)_{1} / \ln \left(w / w_{\mathrm{s}}\right)_{2}=\left(h_{1} / h_{3}\right)^{1 / y},
$$

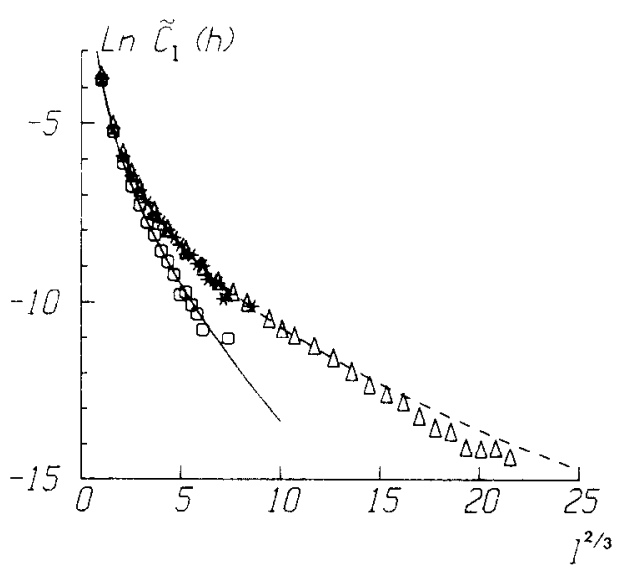

Fig. 6. In $\tilde{c}_{l}(h)$ as defined in eq. (6.11) is plotted here versus $l^{2 / 3}$ in order to show the validity of eq. (6.10). The solid line is the same as the theoretical one in fig. 5 for $P_{2}\left(0.78 T_{c}, h=0\right)$. The dashed line is the theoretical one in fig. 5 for $P_{3}\left(0.89 T_{\mathrm{c}}, h=0\right)$. The data points correspond to $P_{4}$ (triangles), $P_{5}$ (asterisks) and $P_{6}$ (circles). 
which relates fugacities and magnetic fields at two different temperatures when $h$ is small enough. As a further test of scaling behavior we find that eq. (6.12) holds indeed with $y \approx 0.45$ in the case of our data at points $P_{4}, P_{5}$ and $P_{6}$ where $h<0.1$. This fact was made evident in fig. 3 where, together with the data and a polynomic fit to it, we included the behavior $\mathrm{e}^{-h t}$ which is implied by the classical theory (see eq. (8.2)). One should be warned, however, that the data analysed in ref. 20 (which was kindly provided to us by D. Stauffer) corresponding to $0.2<|h|<0.6$ implies $y$ close to 1 in eq. (6.12), a fact which should be expected in the case of "strong" magnetic fields according to a theorem by Souillard and Imbrie ${ }^{17}$ ), see ref. 23 .

\section{Clusters energy}

Eq. (1.5) defines the cluster "energy" $s_{l}$, i.e. the mean number of surface and interior bonds incident on a cluster of size $l$. The scaling assumption (6.1) also leads to some definite predictions about the behavior of $s_{l}$ with $l$ which can be checked against computer simulation data and exact results. In particular, using (6.1) in definition (1.5) one readily has

$$
s_{l}=l^{x} \int u f_{1}\left(\epsilon l^{z}, h l^{y}, u\right) \mathrm{d} u / \int f_{1}\left(\epsilon l^{z}, h l^{y}, u\right) \mathrm{d} u,
$$

after making an obvious change of variables. Assuming again the factorization of $f_{1}, f_{1}\left(\epsilon l^{z}, h l^{y}, u\right)=f_{2}\left(\epsilon l^{z}, h l^{y}\right) f_{\mathrm{s}}(u)$, we obtain

$$
s_{l}=\text { constant } \times l^{x}, \quad x=0.84 \pm 0.01,
$$

where the value for $x$ follows from (6.3) and (6.5).

We find that our data at any temperature and external magnetic field $\left(P_{1}-P_{6}\right)$ can be represented quite well by

$$
s_{l} \sim l^{0.86+0.03}
$$

as shown by fig. 7 in accordance with (7.2). This is also consistent with the familiar assumption that $s_{l} \sim l^{x}, 2 / 3 \leqslant x \leqslant 1$. On the other hand, the consideration of density differences between the cluster and its surrounding medium leads to the prediction $^{19}$ ) that the surface energy should be proportional to $l^{1 / \beta \delta+1 / \delta}=l^{0.84}$, in fair accordance with (7.2) and (7.3); in fact comparing this prediction with (6.3) one obtains $y \approx 4 / 9$, consistent with (6.5).

We also mention that avoiding the factorization of $f_{1}$ one is led to $s_{l}=l^{x} f\left(\epsilon l^{2}\right)$, a formula which Binder tried to verify ${ }^{5}$ ), while our data seem to imply a constant function $f$, as in eq. (7.2). This, however, might perhaps not hold any more for sizes, magnetic fields or temperatures outside the ranges discussed here. In fact one 


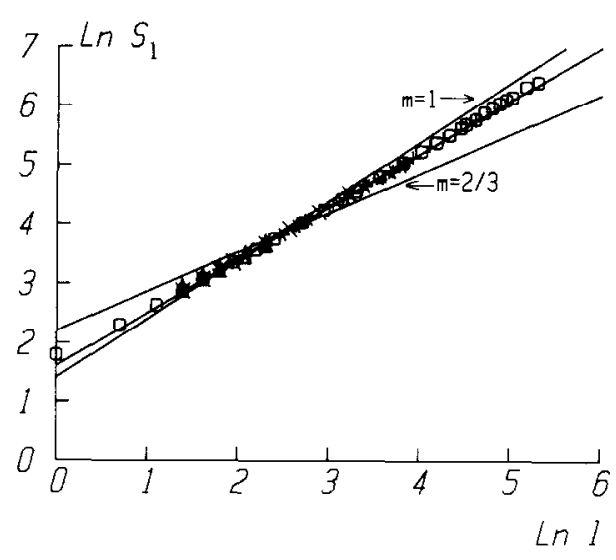

Fig. 7. A plot of the data $s_{l}$ versus $l$ corresponding to the phase points $P_{1}$ (triangles) $P_{2}$ (asterisks), $P_{3}$ (circles), $P_{4}$ (plusses), $P_{5}$ (crosses) and $P_{6}$ (dashed lines) to show that $s_{i} \sim 1^{0.86}$. The lines $\sim 1^{2 / 3}$ and $\sim l$ are also shown. The shown behavior is also consistent with $s_{l} \sim l+\alpha l^{2 / 3}{ }^{23}$ ).

may detect some temperature dependence far from $T_{\mathrm{c}}$ in the exponent $x$ when assuming $s_{l} \sim l^{x}$ (see also the end of section 6).

In order to show up this fact we have fitted the exact result (4.8) for $3 \leqslant l \leqslant 10$ (note that $s_{1}=6, s_{2}=10, s_{3}=14$, independent of temperature) to the eq. (7.2). We thus find that $x=0.63,0.84,0.86,0.87$ and 0.897 describe very well the data at $T / T_{\mathrm{c}}=0,0.6,0.8,0.9$ and $\infty$, respectively, in the assumption (7.2). The latter value for $x$ suggests to look also for a linear behavior at infinite temperature. We thus find that the exact values (4.8) at $T=\infty$ can also be fitted by $s_{t}=2.42+3.88 l$, $1 \leqslant l \leqslant 10$ (with a correlation coefficient equal to 0.99996 ). This linear behavior, which was already reported in ref. 21 as a consequence of computer data at infinite temperature (outside the percolation region, $\rho<0.31$ ) for larger values of $l$, is seen to be clearly inadequate to describe the exact values (4.8) at, say $T=0 \mathrm{~K}$.

We have also tried to fit the data to a behavior $s_{l}=\alpha_{1} l+\alpha_{2} l^{2 / 3}$ as suggested from the previous result and the work in ref. 22 ; it then follows $s_{l} l^{-2 / 3}=2.73+2.69 l^{1 / 3}$ from our combined data at $0.6 \leqslant T / T_{c} \leqslant 0.9$. This is a fit slightly worse than (7.3) and clearly inadequate to describe the exact results (4.8) at $T=\infty$, but it seems definitely consistent with our data at finite temperature. Note that a behavior $s_{l} \sim l+\alpha l^{2 / 3}$ follows from eq. (7.1) when the function $f_{l}$ does not factorize.

\section{Conclusions}

A three-dimensional Ising model on a simple cubic lattice with 125000 sites, periodic boundary conditions and a nearest-neighbor interaction favoring phase 
segregation was left to evolve towards equilibrium at low, constant densities (Kawasaki dynamics) ${ }^{7}$ ). We have analysed the equilibrium cluster distribution at different (small) external magnetic fields and different. temperatures, $0.6 \leqslant T / T_{\mathrm{c}} \leqslant 0.9$, along the coexistence line and in the one-phase region, far from the percolative phase transition.

\subsection{Scaling}

The data suggest (figs. 2-7 and tables $\mathrm{V}-\mathrm{X}$ ) that the equilibrium distribution of sizes $l$ and energies $s, c(l, s)$, scales with temperature and external magnetic field ${ }^{5}$ ), as in eqs. (6.1) and (6.3), with $y=0.45 \pm 0.02$. Assuming the validity of the Fisher droplet model ${ }^{4}$ ) near $T_{\mathrm{c}}$ one is lead to $y=1$; an analysis by MüllerKrumbhaar and Stoll ${ }^{6}$ ), based on much more limited data (near $T_{\mathrm{c}}$ ), gave $y \approx 0.5$. The data we used are also limited and not close enough to $T_{\mathrm{c}}$ so that more experiments and theory would be needed in order to precise the above result. It seems, however, from the analysis in this paper that the real equilibrium cluster distributions have a change with temperature such that even temperatures far from $T_{\mathrm{c}}$ (e.g. $T=0.8 T_{\mathrm{c}}$ ) can be used for the moment with some confidence to compute the exponent $y$. In any case our data seem clearly "prefer" Binder's droplet ideas ${ }^{5}$ ), with a cluster effective size $l^{y}$, to the classical nucleation theory (even at temperatures as low as $T=0.59 T_{\mathrm{c}}$ ): every conclusion in this paper supports consistently the presence of scaling for $T / T_{\mathrm{c}} \geqslant 0.8$ and $h<0.1$.

\subsection{Clusters energy}

The above scaling implies that the mean number of incident (interior and exterior) bonds on a cluster of size $l$ behaves as $s_{l} \sim l^{x}$ with $x \approx 0.84$ which is also consistent with our data for $s_{l}$. In fact we find (fig. 7) $x=0.86 \pm 0.03$ roughly independent of temperature (and small magnetic field) for $0.6 \leqslant T / T_{\mathrm{c}} \leqslant 0.9$ and the size range $1 \leqslant l \leqslant 300$.

We have also computed the exact values $s_{l}$ for $1 \leqslant l \leqslant 10$. These are in agreement with the above result but they also show that there is a crossover (for $l \leqslant 10$ ) from $x \approx 0.6$ at $T=0 \mathrm{~K}$ to $s_{l} \sim a+l, a=$ constant, at $T=\infty$; the latter linear behavior was also reported in the analysis of some computer data at infinite temperature outside the percolation region ${ }^{2}$ ). Our data, on the other hand, show no indication of a behavior $l^{2 / 3}$ for "large" clusters at finite temperature ${ }^{22}$ ). The data, however, are also consistent with the behavior $s_{l}=\alpha_{1} l+\alpha_{2} l^{2 / 3}$ except in the case of the exact results, $l \leqslant 10$, at $T=\infty^{23}$ ).

\subsection{Classical nucleation theory}

The Fisher droplet model (2.1) with conventional parameters ${ }^{46}$ ) cannot fit the 
data. Instead eq. (2.3) with a temperature-dependent exponent $\tau$ gives a reasonable fit to the data. This fit, which is good enough at $T=0.59 T_{\mathrm{c}}$, becomes worse with increasing temperature. When comparing that equation with the exact values for $c_{l}$ we find that the fit also becomes worse with descreasing temperatures, and that $\tau(T)$ decreases with temperature changing to negative values at $T \leqslant 0.4 T_{\text {c }}$ (see table III). This temperature-dependence on $\tau$ is inconsistent with the main assumptions leading to the simple droplet $\operatorname{model}^{4}$ ) and with the extrapolation of this model to the neighborhood of $T_{\mathrm{c}}$. Moreover the resulting equation contains then too many parameters so that we find surprisingly that it cannot provide a better fit to the data.

It seems to us that the approximate validity of the classical theory around $T \approx 0.6 T_{\mathrm{c}}(h=0)$ is rather an accident. As a matter of fact the results of a series of Monte Carlo experiments at $T=0.59 T_{\mathrm{c}}{ }^{20,23}$ ) show also (slight) deviations from classical theory. Of course, our description (2.4) recovers the classical results when $l \rightarrow \infty$, in accordance with recent approaches ${ }^{24}$ ).

Concerning the dependence on the field, the classical nucleation theory predicts that

$$
c_{l}=c_{0}^{\prime}(h, T) \exp \left[-a l^{2 / 3}-h l\right]
$$

for "large" clusters and "low" temperatures. Here $a=a(T)=4 \pi R^{2} \gamma / k T l^{2 / 3}$, where $\gamma$ is the bulk surface tension (domain wall energy) and $R$ is the cluster radius; assuming $4 \pi R^{3} / 3=l$ one has $a(T)=(36 \pi)^{1 / 3} \gamma / k T$. Thus, the prediction is that a plot $\ln c_{l}$ versus $l^{2 / 3}$ should give straight lines (at large $l$ ) with a slope independent of the field $h$ at a given temperature.

This is in general not supported by our data. In fact eq. (8.1) implies

$$
c_{l /} / c_{l}^{\mathrm{s}}=\left[c_{0}^{\prime}(h, T) / c_{0}^{\prime}(o, T)\right] \mathrm{e}^{-h l} .
$$

Instead (fig. 3) we have eq. (6.7) in the case of small magnetic fields $(h<0.1)$ or eq. (6.10) which is valid for any value of the field. The relation between eqs. (6.7) and (6.10) is given by eq. (6.12) which implies $y \approx 0.45$ in the case of the data analysed in this paper while it seems to give $y \approx 1$ when the magnetic field is stronger ${ }^{20,23}$ ).

\subsection{Modified droplet model}

The above facts justify to look for a more general description of the equilibrium cluster distribution. We have thus introduced eq. (2.4) as a semiphenomenological modification of classical nucleation theory. Requiring $\tau=2.2$ in eq. (2.4), as in the Fisher droplet $\operatorname{model}^{4}$ ), one obtains a very good description of the data (fig. 1). Some results of the present analysis (figs. 2-4 and 7), however, imply the use of $\tau=2.09$ which does not introduce important numerical differences. The 
agreement between the data and eq. (2.4) with $\tau=2.09$ is very good for any value of $l$ in the range of temperatures considered here (figs 5 and 6). The proposed description has the advantage over the previous ones that it produces a better fit with less adjustable parameters, that it can be extrapolated (see fig. 4 and eqs. (6.9)) to $T_{\mathfrak{c}}$ (where, however, its validity may not be checked, due to percolation effects, if one maintains our definition of clusters in section 1) and that it incorporates the above facts about scaling. In fact we observe that the description eq. (2.4) becomes better when increasing the temperature from $T=0.59 T_{\mathrm{c}}$ onwards.

\subsection{Renormalized fugacity and partition functions}

The data can also be described in terms of a cluster partition function, $Q_{l}$, and a system renormalized fugacity, $w=w(T, \rho)$, without any restriction on the values of the temperature or magnetic field. We find eqs. (4.1) and (4.3) (with $w$ given in table IV) which slightly improve the approach by Penrose et al. ${ }^{2}$ ) when $l \leqslant 10$. The functions $Q_{l}$ are only exactly known up to $l=10^{16}$ ) but our description, eq. (2.4), allows to extrapolate $Q_{1}-Q_{10}$ to larger values of $l$ (see eqs. (5.1)-(5.3)). The agreement between this alternative approach and the computer data analysed in this paper turns out to be also excellent (tables $\mathrm{V}-\mathrm{X}$ ) this fact stresses the utility and validity of the description based on eq. (2.4).

\section{Acknowledgements}

We thank O. Penrose and D. Stauffer for their valuable comments on a preliminary version of this paper, the latter also for a critical reading of the final manuscript, and D.W. Heermann and D. Stauffer for details about their work in ref. 20 before publication.

\section{References}

1) See, for instance, H. Müller-Krumbhaar, in: Monte Carlo Methods in Statistical Physics, K. Binder, ed. (Springer-Verlag, Berlin, 1979).

2) M.H. Kalos, J.L. Lebowitz, O. Penrose and A. Sur, J. Stat. Phys. 18 (1978) 39.

3) O. Penrose, J.L. Lebowitz, J. Marro, M.H. Kalos and A. Sur, J. Stat. Phys. 19 (1978) 243. O. Penrose et al., to be published.

4) M.E. Fisher, Physics 3 (1967) 255.

5) K. Binder, Ann. Phys. (NY) 98 (1976) 390.

6) H. Müller-Krumbhaar and E.P. Stoll, J. Chem. Phys. 65 (1976) 4294.

7) J. Marro, A.B. Bortz, M.H. Kalos and J.L. Lebowitz, Phys. Rev. B12 (1975) 2000.

J. Marro, J.L. Lebowitz and M.H. Kalos, Phys. Rev. Lett. 43 (1979) 282. 
J.L. Lebowitz, J. Marro and M.H. Kalos, Acta Met. 30 (1982) 297.

8) J.W. Essam and M. Fisher, J. Chem. Phys. 38 (1963) 802.

9) R. Toral, Tesina, Universidad de Barcelona (1982) unpublished.

10) H. Müller-Krumbhaar, Phys. Lett. A50 (1974) 27.

M.F. Sykes, D.S. Gaunt, J. Phys. A 9 (1975) 2131

11) H. Kunz and B. Souillard, Phys. Rev. Iett. 40 (1978) 133; J. Stat. Phys. 19 (1978) 77.

12) E. Stoll, K. Binder and T. Schneider, Phys. Rev. B6 (1972) 2777.

13) See e.g. L. Reatto, Phys. Lett. A33 (1970) 519.

14) C.S. Kiang and D. Stauffer, Z. Phys. 235 (1970) 130.

15) J.L. Lebowitz and O. Penrose, J. Stat. Phys. 16 (1977) 321.

16) M. Sykes, unpublished (1975); see refs. 2, 3.

17) B. Souillard and J. Imbrie, private communication by D. Stauffer; see also, J. Kertész et al. in ref. 23.

18) D.S. Gaunt and C. Domb, J. Phys. C 3 (1970) 1442.

Also the articles by P.G. Watson and by D. Comb, in: Phase Transitions and Critical Phenomena, vol. 3, C. Domb and M.S. Green, eds. (Academic Press, New York, London, 1972).

19) L.P. Kadanoff, in: Proc. Intern. School of Physics Enrico Fermi, Critical Phenomena, M.S. Green, ed. (Academic Press, New York, London, 1971).

D. Stauffer, C.S. Kiang and G.H. Walker, J. Stat. Phys. 3 (1971) 323.

20) D. Stauffer, A Coniglio and D.W. Heermann, Phys. Rev. Lett. 49 (1982) 1299.

D.W. Heermann, A. Coniglio, W. Klein and D. Stauffer, J. Stat. Phys. (in preparation).

21) A. Sur, J.I. Lehowitz, I. Marro, M.H. Kalos and S. Kirkpatrick, J. Stat. Phys. 15 (1976) 345.

22) K. Binder and D. Stauffer, J. Stat. Phys. 6 (1972) 49.

23) J. Marro and R. Toral, in: Phase Transformations in Solids, Materials Research Society Series, A.W. Keneth, ed. (Elsevier, New York) to appear.

24) G. Jacucci, A. Perini and G. Martin, J. Phys. A 16 (1983) 369 